\title{
Systemische Immunsuppressiva in der Therapie der Psoriasis
}

\author{
Systemic Immunosuppressive Drugs in the Treatment of Psoriasis
}

\section{Zusammenfassung}

Immunsuppressiva wirken auf einen Schenkel der psoriatischen Pathogenese, dem aktivierten Immunsystem. Hierbei lassen sich aufgrund ihres Wirkungsprinzips fünf therapeutische Ansatzpunkte erkennen: (i) Hemmung der Effektorzytokine, (ii) Hemmung der T-Zellproliferation, (iii) Hemmung der T-Zellaktivierung, (iv) Hemmung der T-Zellmigration und (v) Modulation der Immunantwort. Die Entwicklung systemischer Immunsuppressiva hat nicht nur das Spektrum der Psoriasistherapie maßgeblich erweitert, sondern hat auch einen profunden Beitrag zum Verständnis der Pathogenese der Psoriasis geleistet. Sie sind sehr wirksam in der Vermeidung von TransplantatabstoBungsreaktionen. Betrachtet man ihr Wirkungs-Nebenwirkungs-Verhältnis hinsichtlich der Behandlung einer benignen chronischen Dermatose, wie der Psoriasis, so muss eine kritische Abwägung bezüglich ihres Einsatzes vorgenommen werden. Systemische Immunsuppressiva müssen demnach schweren, therapierefraktären Fällen der Psoriasis vorbehalten bleiben.

\section{Abstract}

Immunosuppressive drugs act on one part of the psoriatic pathogenesis, the activated immune system. Different therapeutical strategies can be considered on the background of their molecular mechanisms therefore of action: (i) inhibition of effector cytokines, (ii) inhibition of T-cell proliferation, (iii) inhibition of T-cell activation, (iv) inhibition of T-cell migration and (v) modulation of the immunoreaction. On the one hand the development of systemic immunosuppressive drugs has enlarged our therapeutical possibilities in psoriasis. On the other hand these substances increased our understanding of the molecular pathways involved in the pathogenesis of psoriasis. Immunosuppressive drugs are highly potent in avoiding graft reactions, but in therapy of a benign, chronic dermatosis like psoriasis it should be considered that these compounds are also associated with significant cutaneous and/or systemic adverse effects and the risk/benefit ratio must be taken into account. Systemic immunosuppressive drugs are therefore limited to patients with a severe psoriasis refractory to other therapies.

\section{Einleitung}

Die Psoriasis vulgaris ist eine relativ häufige dispositionelle Hautkrankheit, die mit chronisch-entzündlichen Veränderungen der Dermis und Fehldifferenzierung der Epidermis einhergeht. Ihr klinisches Erscheinungsbild und ihre Ausprägung können sowohl individuell als auch im Verlauf stark variieren. Eine Therapie muss sich demnach an dem aktuellen klinischen Bild orientieren und individuell angepasst sein. Das zur Verfügung stehende therapeutische Spektrum reicht von der keratolytischen und sonstigen Lokaltherapie bis hin zur systemischen Immunsuppression. Allgemein lassen sich Therapeutika unterscheiden, die vorwiegend auf die epidermale Differenzierungsstörung abzielen, von denen, die vorwiegend gegen die dermale Entzündungsreaktion gerichtet sind. Diese unterschiedlichen Ansatzpunkte der Therapie spiegeln letztlich die unterschiedlichen Fassetten der Pathogenese der Psoriasis wider. Betrachtet man die therapeutische Wirksamkeit von Immunsuppressiva in der Behandlung der Psoriasis, so konzentriert man sich auf einen Schenkel 
der psoriatischen Pathogenese, dem aktivierten Immunsystem $[1,2]$.

Zum Verständnis der Wirkungsprinzipien von Immunsuppressiva in der Therapie der Psoriasis ist es notwendig, die kutanen immunologischen Vorgänge zu betrachten:

Während in der nicht betroffenen, normalen Haut naive T-Lymphozyten zwischen Blut und Lymphknoten zirkulieren ohne in die Dermis und Epidermis einzudringen, kommt es bei der Entwicklung einer Immunantwort zur Aktivierung von immunkompetenten Zellen, die beide Kompartimente der Haut infiltrieren. Der erste Schritt ist hierbei die Antigen-stimulierte Aktivierung von Antigen-präsentierenden Zellen, d.h. in der Epidermis der Langerhans-Zellen und in der Dermis der dermalen dendritischen Zellen. Sie wandern nach ihrer Aktivierung in die entsprechenden drägnierenden Lymphknoten, wo sie Antigenfragmente den T-Lymphozyten präsentieren und sie so aktivieren. Aktivierung bedeutet in diesem Zusammenhang die klonale Vermehrung von T-Lymphozyten, die gegen das präsentierte Antigen gerichtet sind. Da bei einer Immunreaktion mehrere Antigenfragmente präsentiert werden, entsteht eine Vermehrung mehrerer T-Zellklone, also eine polyklonale Immunantwort. Durch Expression von spezifischen Zelloberflächenmolekülen erhalten die aktivierten T-Lymphozyten eine Adresse, d.h. den Ort, an dem sie benötigt werden. Diese so genannten „Homing“-Signale sind im Falle eines kutanen Immunprozesses die kutanen Lymphozytenassozierten Antigene (engl. CLA). Die so adressierten Immunzellen verlassen den Lymphknoten und gelangen über den Blutweg in den kutanen Gefäßplexus, wo sie mit ihren Homing-Signalen an den entsprechenden Rezeptoren (E-Selektin) binden und das Gefäß verlassen können. Indirekt durch die Sekretion von Zytokinen durch T-Lymphozyten oder direkt durch Bindung an Keratinozyten kommt es zur gesteigerten keratinozytären Proliferation, zur Bildung inflammatorischer Zytokine wie beispielsweise Interleukin 8 (IL-8) durch Keratinozyten sowie zur Sekretion von VEGF (Vascular Endothelial Growth Factor) mit konsekutiver Angiogenese $[3,4]$.

Dieses Szenario eröffnet an verschiedenen Stellen die Möglichkeit eines therapeutischen Eingreifens. Da die Differenzierung der Antigen-präsentierenden Zellen nach Antigenkontakt nicht verhindert werden kann, konzentriert sich das Interesse einer therapeutischen Intervention auf die nachfolgenden Schritte.

Die heute zur Verfügung stehenden Immunsuppressiva sowie die sich in der Entwicklung befindlichen immunsuppressiven Wirkstoffe lassen sich aufgrund ihres Wirkungsprinzips in fünf Gruppen einteilen:

- Hemmung der Effektorzytokine

- Hemmung der T-Zellproliferation

- Hemmung der T-Zellaktivierung

- Hemmung der T-Zellmigration

- Modulation der Immunantwort

Die in der systemischen immunsuppressiven Therapie der Psoriasis eingesetzten bzw. möglicherweise einsetzbaren Wirkstoffe werden im Folgenden hinsichtlich ihrer Hauptwirkung nach dieser Einteilung zusammengefasst. Weitere Fassetten ihrer Wirkung werden dabei bewusst vernachlässigt.

\section{Hemmung der Effektorzytokine}

\section{Cyclosporin A (Sandimmun ${ }^{\circledR}$ )}

Cyclosporin A ist ein sehr lipophiles, zyklisches Oligopeptid mit starker immunsuppressiver Wirkung. Es bindet an das zytosolische Bindungsprotein Cyclophilin, einem Mitglied der Immunophilin-Proteinfamilie. Der Komplex aus Cyclosporin A und Cyclophilin hemmt über die Phosphatase Calcineurin besonders in T-Lymphozyten den Transkriptionsfaktor NF-AT („nuclear factor of activated T cells“). Er ist vor allem für die Ablesung der Genabschnitte verantwortlich, die für Zytokine wie Interleukin 2, Interleukin 6 oder Interferon $\gamma$ kodieren. Es kommt in der Folge zu einer verminderten Biosynthese und Sekretion dieser proinflammatorischen, T-Zell-aktivierenden Proteine. Cyclosporin A unterbindet so die auto- und parakrine Stimulation von T-Lymphozyten. Als immunsuppressiver Therapieansatz wurde Cyclosporin A zuerst zur Vermeidung von Abstoßungsreaktionen bei Nieren-, Herz- und Lebertransplantationen eingesetzt. Sein therapeutischer Effekt in der Behandlung der Psoriasis wurde erstmals 1979 beschrieben. Seither hat eine Reihe weiterer Berichte und Studien seine therapeutische Wirksamkeit klar belegt. Ansprechraten von etwa $80 \%$ und ein Wirkungseintritt nach ca. 2 Wochen mit einem Maximum des therapeutischen Effektes nach 8-12 Wochen sind zu erwarten. Initial werden täglich 2,5 bis $3 \mathrm{mg}$ Cyclosporin A/kg Körpergewicht auf zwei Tagesdosen verteilt, verabreicht; bei ungenügendem Ansprechen kann eine Dosisanpassung bis täglich $5 \mathrm{mg} / \mathrm{kg}$ Körpergewicht erfolgen. Nach erzieltem Therapieerfolg sollte Cyclosporin A nicht abrupt abgesetzt werden, um ein schnelles Rezidiv zu vermeiden, sondern schrittweise reduziert werden. Der guten therapeutischen Wirksamkeit von Cyclosporin A stehen seine potenziellen $\mathrm{Ne}$ benwirkungen gegenüber. Insbesondere eine arterielle Hypertonie sowie eine Nierenfunktionsstörung wird mit einer Häufigkeit von jeweils ca. $20 \%$ angegeben. Dies beschränkt den Einsatz von Cyclosporin A zur Therapie der Psoriasis auf selektionierte, therapierefraktäre Patienten [6-8].

\section{Tacrolimus (Prograf ${ }^{\circledR}$ )}

Tacrolimus (FK 506) gehört in die Gruppe der immunmodulierenden Makrolactame. Obwohl ihre chemische Struktur keine Ähnlichkeit zu Cyclosporin A aufweist, wirken sie auf zellulärer Ebene ähnlich wie Cyclosporin A über die Bindung an Immunophiline. Tacrolimus bindet an das Immunophilin FKBP-12, auch als Makrophilin 12 bezeichnet. Hierdurch wird, wie durch Cyclosporin A, die Biosynthese von proinflammatorischen Mediatoren, wie beispielsweise Interleukin 2, Interleukin 6 oder Tumornekrosefaktor $\alpha$ bewirkt. Eine erfolgreiche systemische Therapie der Psoriasis mit Tacrolimus wurde für eine Dosierung von täglich 0,05-0,15 mg/kg Körpergewicht beschrieben. Das Nebenwirkungsprofil entspricht in etwa dem von Cyclosporin A [9]. Bemerkenswerterweise scheint Cyclosporin A eine stärkere antipsoriatische Wirkung als Tacrolimus zu entfalten, eine Beobachtung, deren Bedeutung bis heute unklar bleibt.

\section{Infliximab (Remicade $\left.{ }^{\circledR}\right)$}

Dieser chimere monoklonale Antikörper gegen Tumornekrosefaktor- $\alpha$ fängt den freigesetzten Entzündungsmediator TNF- $\alpha$ ab und hemmt somit allgemein Entzündungsreaktionen. Infliximab wurde deshalb auch bereits erfolgreich bei entzündlichen Darmund Gelenkerkrankungen eingesetzt. Erhöhte TNF- $\alpha$-Spiegel las- 
sen sich sowohl in psoriatischen Läsionen der Haut als auch in der Synovia bei der Psoriasisarthritis nachweisen. Dies deutet die prinzipielle Möglichkeit der Wirksamkeit bei Psoriasispatienten an. In einer kürzlich veröffentlichten doppelblinden, randomisierten Studie an 33 Patienten wurden dabei Plazebo mit $5 \mathrm{mg} / \mathrm{kg}$ bzw. $10 \mathrm{mg} / \mathrm{kg}$ Körpergewicht als intravenöse Infusion in Woche 0, 2 und 6 verabreicht, verglichen. Dabei zeigte sich ein Wirkungseintritt nach ca. 4 Wochen und bei $82 \%$ der mit Infliximab behandelten Patienten eine Reduktion des PASI-Scores um $82 \%$ nach 10 Wochen. Als rezidivfreie Zeit werden 8 Monate angegeben. Eine Kombination von Infliximab und Methotrexat zur Psoriasistherapie wurde beschrieben. TNF- $\alpha$ nimmt eine zentrale Rolle in Entzündungs- und Infektionsprozessen ein. Insofern verwundert es nicht, dass eine Blockade von TNF- $\alpha$ mit einem erhöhten Infektionsrisiko einhergeht. So wurde die Reaktivierung einer Tuberkulose kürzlich berichtet [10-13].

\section{Etanercept (Enbrel ${ }^{\circledR}$ )}

Etanercept greift ebenfalls am Entzündungsmediator TNF- $\alpha$ an, allerdings handelt es sich um ein TNF- $\alpha$-Rezeptor-Immunglobulinfusionsprotein, das TNF- $\alpha$ neutralisiert. Auch hier liegen umfangreichere Daten zur Behandlung der Rheumatoidarthritis und des Morbus Crohn vor. In einer randomisierten Studie wurden 30 Patienten mit Psoriasis zweimal wöchentlich mit $25 \mathrm{mg}$ Etanercept als subkutane Injektion über insgesamt 12 Wochen behandelt und mit 30 plazebobehandelten Patienten verglichen. Es zeigte sich eine Besserung der Psoriasis bei 87\% der Etanercept-behandelten Patienten gegenüber $13 \%$ in der Plazebokontrolle, wobei eine $75 \%$ ige Reduktion des PASI bei 34\% der Etanercept-behandelten Patienten auftrat. Als rezidivfreie Zeit werden 6 Monate angegeben. Weiterhin sind einige positive Fallberichte in der letzten Zeit zum Einsatz von Etanercept in der Therapie der Psoriasis und psoriatischen Arthritis erschienen. Hinsichtlich des Nebenwirkungsprofiles gelten die gleichen Angaben wie bei Infliximab [14-16].

\section{ABX-IL-8}

Dieser humane anti-Interleukin-8-Antikörper neutralisiert dieses, in der Psoriasispathogenese beteiligte Chemokin. Bisher existieren nur experimentelle Daten. Klinische Studien zur Therapie der Psoriasis liegen bisher nicht vor. Ob die Neutralisation von IL-8 im oberen Stratum spinosum gelingt ist fraglich, da dieser Bereich der Epidermis für die neutralisierenden Antikörper möglicherweise schlecht zugänglich ist. Insgesamt müssen weitere experimentelle und klinische Ergebnisse abgewartet werden, um Erfahrungen mit dieser möglichen Therapiestrategie zu sammeln [17].

\section{Hemmung der T-Zellproliferation}

\section{Methotrexat}

Methotrexat (MTX) ist ein synthetisches Dihydrofolat-Analogon, das als Antimetabolit die Bildung von Tetrahydrofolsäure hemmt und somit eine Reihe Folsäure-abhängiger zellulärer Reaktionen blockiert. Hiervon betroffen ist u.a. die Biosynthese von Nukleinsäuren, Membranphospholipiden und Proteinen. Somit führt MTX zur Hemmung der Zellteilung und beeinflusst besonders Zellen, die sich in der S-Phase des Zellzyklus befinden. Neben dieser zelltyp-unspezifischen zytostatischen Wirkung, die so- wohl Keratinozyten, T-Lymphozyten sowie Langerhans-Zellen erfasst, konnte im Niedrigdosisbereich auch eine verminderte Freisetzung von Effektorzytokinen, wie IL-1 $\beta$ oder IL-6 sowie eine Hemmung der Chemotaxis neutrophiler Granulozyten beobachtet werden. MTX entfaltet somit neben seiner antiproliferativen Wirkung auf Keratinozyten auch eine nicht unerhebliche antiinflammatorische und immunsuppressive Wirkung. Insgesamt steht mit MTX ein kostengünstiger Wirkstoff mit sehr gutem therapeutischen Effekt zur Verfügung. Aufgrund der jahrzehntelangen Erfahrung mit diesem Medikament ist das Wirkungs-/Nebenwirkungsspektrum umfassend bekannt und macht einen sicheren klinischen Einsatz möglich $[18,19]$.

\section{Mycophenolatmofetil (CellCept ${ }^{\circledR}$ )}

Mycophenolatmofetil gehört zur Gruppe der so genannten Prodrugs; der aktive Metabolit ist die Mycophenolsäure. Dieser Morpholinoethylester hat eine sehr gute Bioverfügbarkeit und wird nach oraler Aufnahme zu 94\% resorbiert und vollständig zu seinem aktiven Metaboliten Mycophenolsäure hydrolysiert. Sie hemmt nicht-kompetitiv und reversibel das Enzym Inosinmonophosphat-Dehydrogenase und damit den De-novo-Biosyntheseweg von Guanosinmonophosphat (GMP). GMP wird zur Nukleinsäure-Biosynthese benötigt und spielt u.a. eine Rolle bei Glycosylierungsschritten von Zelloberflächenmolekülen. Während die meisten Zellen diese Hemmung über einen weiteren Syntheseweg kompensieren können, ist in B- und T-Lymphozyten nur dieser De-novo-Biosyntheseweg möglich; hierdurch ergibt sich die B-/T-Zelltypspezifizität der antiproliferativen Wirkung von Mycophenolsäure. Mycophenolatmofetil ist derzeit nur als Immunsuppressivum zur Vermeidung von Abstoßungsreaktionen bei Patienten mit Nieren- oder Herztransplantationen zugelassen. Zur erfolgreichen, systemischen Behandlung mit Mycophenolatmofetil von Patienten mit Psoriasis liegt bereits eine Reihe von Berichten vor. Hierbei müssen auch die früheren, erfolgreichen Studien mit Mycophenolsäure erwähnt werden. Mycophenolatmofetil entfaltet eine gute antipsoriatische Wirkung in einer Dosierung von $2 \times 1 \mathrm{~g} / \mathrm{d}$. Häufige Nebenwirkungen sind hierbei gastrointestinale Beschwerden, ferner werden ein erhöhtes Infektionsrisiko sowie Leukopenien beschrieben. Da der aktive Metabolit, die Mycophenolsäure zu 97\% an Plasmaalbumin gebunden vorliegt, müssen bei Kombinationstherapien mit anderen Medikamenten mit hoher Plasmaalbuminbindung, wie beispielsweise Retinoiden, Dosisanpassungen vorgenommen werden. Insgesamt stellt die systemische Gabe von Mycophenolatmofetil eine interessante Alternative für Patienten mit schwerer, therapierefraktiver Psoriasis dar [20-24].

\section{Leflunomid (Arava ${ }^{\circledR}$ )}

Dieses Isoxazolderivat ist ebenfalls ein Prodrug, dessen aktiver Metabolit u.a. die Dihydroorotatdehydrogenase hemmt, einem Schlüsselenzym in der Pyrimidin- und damit Nukleinsäure-Biosynthese. Dieser De-novo-Biosyntheseweg von Pyrimidin ist vermutlich für T- und B-Lymphozyten essenziell, da besonders ihre Proliferation gehemmt wird. Leflunomid entfaltet so eine immunsuppressive Wirkung, die bei der Behandlung der Rheumatoidarthritis einen guten Therapieeffekt zeigt. Zur systemischen Therapie der Psoriasis liegen bisher wenige klinische Daten vor. Kürzlich wurde ein erfolgreicher Einsatz von Leflunomid $(10 \mathrm{mg} / \mathrm{d})$ allerdings in Kombination mit Prednisolon $(10 \mathrm{mg} / \mathrm{d})$ und topisch angewendetem Vitamin- $\mathrm{D}_{3}$-Analogon berichtet. Die 
nachgewiesene teratogene Wirkung von Leflunomid ist beim geplanten therapeutischen Einsatz zu berücksichtigen $[25,26]$.

\section{Daclizumab (Zenapax ${ }^{\circledR}$ )}

Die Proliferation von T-Lymphozyten wird u.a. durch Interleukin-2 durch Bindung an den IL-2-Rezeptor der T-Zellen kontrolliert. Hier greift eine weitere innovative Therapiestrategie an. Durch einen humanisierten Antikörper gegen die $\alpha$-Untereinheit des IL-2-Rezeptors (CD25), Daclizumab, wird diese Signalkaskade blockiert. Dies wurde erfolgreich in der Prävention von Abstoßungsreaktionen nach Nierentransplantation eingesetzt. In einer ersten Studie mit 19 Patienten mit Psoriasis konnte gezeigt werden, dass mit zunehmender Blockade des IL-2-Rezeptors es zur zunehmenden Reduktion der Psoriasis kam. Eine Reduktion der Daclizumab-Dosierung korrelierte mit der erneuten Verschlechterung der Psoriasis. Welchen Stellenwert dieser Therapieansatz bei der Psoriasis zukünftig haben wird, bleibt abzuwarten [27-29].

\section{Basiliximab (Simultect ${ }^{\circledR}$ )}

Auch dieser monoklonale Antikörper greift am IL-2/IL-2-Rezeptor-System an, in dem er am Interleukin-2-Rezeptor bindet. Es wurden in der Transplantationsmedizin bereits größere Erfahrungen mit diesem Antikörper gesammelt. Der Einsatz von Basiliximab zur Therapie der Psoriasis ist in Fallberichten beschrieben worden. Es fehlen aber noch kontrollierte Studien [30].

\section{Denileukin diftitox (Ontak ${ }^{\circledR}$ )}

Schließlich nutzt eine weitere Therapiestrategie das IL2/IL-2-Rezeptor-System auf ganz andere Art. Durch Fusion von humanem IL-2 mit einem Fragment von Diphtherietoxin entsteht ein zytotoxisches Fusionsprotein ( $\mathrm{DAB}_{389} \mathrm{IL}-2$, Denileukin diftitox), das spezifisch über den IL-2-Rezeptor an aktivierte T-Lymphozyten bindet und diese ausschaltet. Hauptsächlich liegen Erfahrungen zum Einsatz von Denileukin diftitox bei kutanen T-Zell-Lymphomen vor. In einer multizentrischen Phase-II-Studie konnte zwar eine generelle antipsoriatische Wirksamkeit gezeigt werden, allerdings führten ausgeprägte grippeähnliche Nebenwirkungen zum Therapieabbruch bei 10 von 41 Patienten [31,32].

\section{Hemmung der T-Zellaktivierung}

Für die indirekte Hemmung der T-Zellaktivierung durch Hemmung der Effektorzytokinfreisetzung gibt es eine Reihe von Wirksubstanzen, die teilweise bereits zur Behandlung der Psoriasis zugelassen sind. Anders sieht es bei Therapeutika aus, die direkt in die T-Zellaktivierung eingreifen; sie befinden sich derzeit noch in der Entwicklung bzw. klinischen Prüfung.

\section{OKTcdr4a}

T-Zellaktivierung durch Antigen-präsentierende Zellen erfolgt u. a. über den T-Zellrezeptor-Komplex, zu dem primär CD3, CD4 und CD8 gehören und weitere kostimulatorische Signale, wie beispielsweise CD80/CD28, CD40/CD40L oder LFA-3/CD2 charakterisiert wurden. OKTcdr4a ist ein humanisierter Antikörper, der spezifisch an CD4 bindet und so CD4-positive, dermale T-Helferzellen angreift. In mehreren Studien konnte bereits die prinzipielle Wirksamkeit dieses Therapieprinzips bei Patienten mit
Psoriasis belegt werden. Phase-III-Studien liegen bisher nicht vor [33].

\section{MEDI-507}

Ähnlich wie OKTcdr4a greift MEDI-507 in die T-Zellaktivierung ein, wobei MEDI-507 das kostimulatorische Signal zwischen LFA-3 der Antigen-präsentierenden Zelle und dem CD2-Rezeptor des T-Lymphozyten blockiert. MEDI-507 ist ein humanisierter monoklonaler Antikörper gegen CD2 und könnte in der Behandlung der Psoriasis erfolgreich sein [34].

\section{Alefacept (Amevive ${ }^{\circledR}$ )}

Auf das kostimulatorische Signal zwischen LFA-3 und CD2 zielt ebenfalls eine weitere Strategie ab. Ein Immunglobulinfusionsprotein, das die Bindungsdomäne von LFA-3 enthält, Alefacept, blockiert durch Bindung an den CD2-Rezeptor CD2-positive T-Memory-Zellen. Vor dem Hintergrund, dass in der psoriatischen Läsion eine Infiltration mit CD2-positiven T-Memory-Zellen nachweisbar ist, wurde eine multizentrische, randomisierte, plazebokontrollierte und doppelblinde Studie initiiert, die eine Verbesserung des PASI nach 12 Wochen um mindestens 75\% bei $28 \%$ der Patienten zeigte, die einmalig 7,5 mg Alefacept intravenös erhalten hatten. Eine weitere Verbesserung ergab sich nach einem zweiten Therapiezyklus $[35,36]$.

\section{Hemmung der T-Zellmigration}

Für diesen Wirkungsmechanismus gibt es derzeit keine zugelassenen Medikamente. Eine Substanz ist allerdings bereits in der klinischen Prüfung.

\section{Efalizumab (Raptiva ${ }^{\circledR}$ )}

Efalizumab ist ein rekombinanter humanisierter monoklonaler Antikörper, der an das Lymphozytenoberflächenantigen CD11a bindet. Bei CD11a (LFA-1) handelt es sich um ein Adhäsionsprotein der Integrin-Familie. Seine Blockierung durch Efalizumab führt zu einer verminderten Bindung von T-Lymphozyten an das Endothel über das Adhäsionsprotein ICAM-1 und verhindert dadurch die Migration von T-Lymphozyten in die Dermis und Epidermis. Darüber hinaus wird auch die Interaktion von bereits in der Dermis und Epidermis befindlicher T-Lymphozyten mit ortsständigen Zellen, wie beispielsweise Keratinozyten unterbunden und so die intraläsionale entzündliche und zytotoxische Reaktion gehemmt. In mehreren plazebokontrollierten, randomisierten, doppelblinden Phase-III-Studien wurde die Wirksamkeit dieses Therapieansatzes untersucht. Bei $41 \%$ der behandelten Patienten zeigte sich nach 12 Wochen eine Verbesserung des PASI um mindestens $75 \%$. Als Wirkungseintritt wurde ein Zeitraum von 2-4 Wochen angegeben und als rezidivfreies Intervall 2-3 Monate. Ein Rebound wurde in ca. 5\% der Patienten beobachtet. Efalizumab wird subkutan einmal wöchentlich $1,0 \mathrm{mg}$ / kg Körpergewicht injiziert. Das bisher bekannte Nebenwirkungsprofil zeigt als sehr häufige Nebenwirkungen grippeähnliche Symptome (Fieber, Schüttelfrost, Kopf- und Muskelschmerzen) sowie eine Leuko- und Lymphozytose; ferner wurden häufig ein Anstieg der Lebertransamidasen und Überempfindlichkeitsreaktionen beobachtet $[37,38]$. 


\section{Efomycine M}

Diese Substanz ist ein Selektininhibitor und verhindert die Interaktion zwischen Lymphozyten und Endothelzellen, die zur Extravasation der Entzündungszellen notwendig ist. Efomycine M ist somit ein Vertreter einer neuen Substanzklasse, die sich für den Einsatz bei entzündlichen Dermatosen, wie der Psoriasis eignen könnte. Klinische Studien oder Fallberichte liegen für diese Verbindung noch nicht vor [39].

\section{Modulation der Immunantwort}

\section{Interleukin 4}

In der Pathogenese der Psoriasis spielen, wie bereits erwähnt, Th1-Zellen und das von ihnen sezernierte Interferon $\gamma$ eine Rolle. Interleukin 4 beeinflusst direkt das Th1/Th2-Gleichgewicht, in dem es eine Th2-Differenzierung anregt. Diese Wirkung wird beim therapeutischen Einsatz zur Behandlung der Psoriasis genutzt. Bisher liegt eine Dosisfindungsstudie vor, die eine gute Verträglichkeit und ein klinisches Ansprechen der behandelten Psoriatiker kürzlich berichtete [40].

\section{Interleukin 10}

Interleukin 10 beeinflusst ebenfalls das Th1/Th2-Gleichgewicht und hemmt die Produktion von Interferon $\gamma$. Diese immunmodulatorische und antiinflammatorische Wirkung bildet die Basis für einen therapeutischen Einsatz in der Therapie der Psoriasis. Bisher liegen nur eine Pilot- und zwei Phase-II-Studien hierzu vor, die bei subkutaner Injektion eine gute Verträglichkeit und ein klinisches Ansprechen bei der Mehrheit der behandelten Psoriatiker berichten. Für eine endgültige Einschätzung ist es derzeit zu früh $[41,42]$.

\section{Interleukin 11 (IL-11)}

Auch Interleukin 11 greift in das Zytokinnetzwerk der Haut ein und bewirkt einen Wechsel vom Th1-Zytokinmuster zum Th2-Zytokinmuster. In einer klinischen Studie konnte durch subkutane Injektion von Interleukin 11 über 8 Wochen eine klinische Besserung bei 11 von 12 Patienten beobachtet werden, die mit einer Reduktion proinflammatorischer Mediatoren wie TNF- $\alpha$, IFN- $\gamma$, IL-8 und IL-1 $\beta$ einherging. Auch hier fehlen bisher Folgestudien, die für eine bessere Einschätzung der therapeutischen Wirksamkeit notwendig sind [43].

\section{Schlussfolgerungen}

Abschließend lässt sich feststellen, dass systemische Immunsuppressiva nicht nur das Spektrum der Psoriasistherapie maßgeblich erweitert haben, sondern auch einen profunden Beitrag zum Verständnis der Pathogenese der Psoriasis geleistet haben. Dieses Wissen stellt nun wiederum die Basis für die Entwicklung neuer, selektiver Therapeutika dar, die an der Schwelle zum klinischen Einsatz stehen. Da die Entwicklung von immunsuppressiv wirksamen Substanzen die Vermeidung von Transplantatabstoßungsreaktionen zum Ziel hatte, fehlt einerseits die Selektivität bezüglich der Pathogenese der Psoriasis, andererseits erklärt sich so das, für eine benigne chronische Dermatose ungünstige Wirkungs-Nebenwirkungs-Verhältnis. Systemische Immunsuppressiva sind demnach schweren, therapierefraktären Fällen der
Psoriasis vorbehalten und sollten nicht aufgrund ihrer vordergründig schnellen und guten Wirkung sowie ihrer bequemen Applikation breit eingesetzt werden. Dabei sollte auch berücksichtigt werden, dass das Rezidiv-freie Intervall nach einer alleinigen Therapie mit einem Immunsuppressivum in der Regel relativ kurz ist, so dass hier Vorsorge getroffen werden muss. Schließlich dürfen auch nicht die anfallenden jährlichen Behandlungskosten unerwähnt bleiben, die bei den zugelassenen Immunsuppressiva bis zu 10000 Euro betragen und bei den neu entwickelten biologischen Wirksubstanzen 25000 Euro übersteigen können. Alle diese Betrachtungen mahnen zum maßvollen Umgang mit dieser hochpotenten und für die Therapie der Psoriasis aussichtsreichen Substanzgruppe.

\section{Literatur}

${ }^{1}$ Christophers E. The immunopathology of psoriasis. Int Arch Allergy Immunol 1996; 110: 199-206

${ }^{2}$ Geilen CC, Orfanos CE. Standard and innovative therapy of psoriasis. Clin Exp Rheumatol 2002; 20 (Suppl 28): 81 - 87

${ }^{3}$ Ellis CN, Krueger GG. Treatment of chronic plaque psoriasis by selective targeting of memory effector T lymphocytes. N Engl J Med 2001; 345: $248-255$

${ }^{4}$ Krueger JG. The immunologic basis for the treatment of psoriasis with new biologic agents. J Am Acad Dermatol 2002; 46: 1 - 23

${ }^{5}$ Müller W, Herrmann B. Cyclosporine A for psoriasis. N Engl J Med 1979; 301: 55

${ }^{6}$ Berth-Jones J, Voorhees JJ. Consensus conference on cyclosporin A microemulsion for psoriasis. Br J Dermatol 1996; 135: 775-777

${ }^{7}$ Mrowietz U, Färber L, Henneicke von Zepelin HH, Bachmann H, Welzel $\mathrm{D}$, Christophers E. Long-term maintenance therapy with cyclosporine and post-treatment survey in severe psoriasis, results of a multicenter study. J Am Acad Dermatol 1995; 33: 470-475

${ }^{8}$ Al-Daraji WI, Grant KR, Ryan K, Saxton A, Reynolds NJ. Localization of calcineurin/NFAT in human skin and psoriasis and inhibition of calcineurin/NFAT activation in human keratinocytes by cyclosporin A. J Invest Dermatol 2002; 118: 779-788

${ }^{9}$ The European FK 506 Multicentre Psoriasis Study Group. Systemic tacrolimus (FK 506) is effective for the treatment of psoriasis in a double-blind, placebo-controlled study. Arch Dermatol 1996; 132: 419-423

${ }^{10} \mathrm{Oh}$ CJ, Das KM, Gottlieb AB. Treatment with anti-tumor necrosis factor alpha (TNF-alpha) monoclonal antibody dramatically decreases the clinical activity of psoriasis lesions. J Am Acad Dermatol 2000; 42: $829-830$

${ }^{11}$ Chaudhari U, Romano P, Mulcahy LD, Dooley LT, Baker DG, Gottlieb AB. Efficacy and safety of infliximab monotherapy for plaque-type psoriasis: a randomised trial. Lancet 2001; 357: $1842-1847$

12 Kirby B, Marsland AM, Carmichael AJ, Griffiths CE. Successful treatment of severe recalcitrant psoriasis with combination infliximab and methotrexate. Clin Exp Dermatol 2001; 26: 27-29

${ }^{13}$ Nunez Martinez O, Ripoll Noiseux C, Carneros Martin JA, Gonzales Lara V, Gregorio Maranon HG. Reactivation tuberculosis in a patient with anti-TNF-alpha treatment. Am J Gastroentererol 2001; 96: $1665-1666$

${ }^{14}$ Mease PJ, Goffe BS, Metz J, VanderStoep A, Finck B, Burge DJ. Etanercept in the treatment of psoriatic arthritis and psoriasis: a randomised trial. Lancet 2000; 356: 385-390

${ }^{15}$ Yazici Y, Erkan D, Lockshin MD. A preliminary study of etanercept in the treatment of severe, resistant psoriatic arthritis. Clin Exp Rheumatol 2000; 18: $732-734$

${ }^{16}$ Iyer S, Yamauchi P, Lowe NJ. Etanercept for severe psoriasis and psoriatic arthritis: observations on combination therapy. Br J Dermatol 2002; 146: $118-121$

17 Huang S, Mills L, Mian B, Tellez C, McCarty M, Yang XD, Gudas JM, BarEli M. Fully humanized neutralizing antibodies to interleukin-8 (ABXIL8) inhibit angiogenesis, tumor growth, and metastasis of human melanoma. Am J Pathol 2002; 161: 125 - 134 
${ }^{18}$ Roenigk HH Jr, Auerbach R, Maibach H, Weinstein G, Lebwohl M. Methotrexate in psoriasis: consensus conference. J Am Acad Dermatol 1998; 38: $478-485$

${ }^{19}$ Kuijpers AL, van de Kerkhof PC. Risk-benefit assessment of methotrexate in the treatment of severe psoriasis. Am J Clin Dermatol 2000; 1 : $27-39$

${ }^{20}$ Geilen CC, Tebbe B, Bartels CG, Krengel S, Orfanos CE. Successful treatment of erythrodermic psoriasis with mycophenolate mofetil. $\mathrm{Br} \mathrm{J}$ Dermatol 1998; 138: 1101 - 1102

${ }^{21}$ Haufs MG, Beissert S, Grabbe S, Schütte B, Luger TA. Psoriasis vulgaris treated successfully with mycophenolate mofetil. Br J Dermatol 1998; 138: $179-181$

22 Geilen CC, Arnold M, Orfanos CE. Mycophenolate mofetil as a systemic antipsoriatic agent: Positive experience in 11 patients. $\mathrm{Br} \mathrm{J}$ Dermatol 2001; 144: $583-586$

${ }^{23}$ Epinette WW, Parker CM, Jones EL, Greist MC. Mycophenolic acid for psoriasis: a review of pharmacology, long-term efficacy, and safty. J Am Acad Dermatol 1987; 17: 962 - 971

${ }^{24}$ Grundmann-Kollmann M, Mooser G, Schraeder P, Zollner T, Kaskel P, Ochsendorf F, Boehncke WH, Kerscher M, Kaufmann R, Peter RU. Treatment of chronic plaque-stage psoriasis and psoriatic arthritis with mycophenolate mofetil. J Am Acad Dermatol 2000; 42: 835-837

${ }^{25} \mathrm{Xu}$ X, Williams JW, Gong $\mathrm{H}$ et al. Two activities of the immunosuppressive metabolite of leflunomide, A77 1726. Inhibition of pyrimidine nucleotide synthesis and protein tyrosine phosphorylation. Biochem Pharmacol 1996; 52: $527-534$

${ }^{26}$ Reich K, Hummel KM, Beckmann I, Mössner R, Neumann C. Treatment of severe psoriasis and psoriatic arthritis with leflunomide. Br J Dermatol 2002; 146: 335-336

${ }^{27}$ Krueger JG, Walters IB, Miyazawa M, Gilleaudeau P, Hakimi J, Light S, Sherr A, Gottlieb AB. Successful in vivo blockade of CD25 (high-affinity interleukin 2 receptor) on T cells by administration of humanized antiTac antibody to patients with psoriasis. J Am Acad Dermatol 2000; 43: $448-458$

${ }^{28}$ Wohlrab J, Fischer M, Taube KM, Marsch WC. Treatment of recalcitrant psoriasis with daclizumab. Br J Dermatol 2001; 144: 209-210

29 Gottlieb AB, Bos JD. Recombinantly engineered human proteins: transforming the treatment of psoriasis. Clin Immunol 2002; 105: $105-116$

${ }^{30}$ Mrowietz U, Zhu K, Christophers E. Treatment of severe psoriasis with anti-CD25 monoclonal antibodies. Arch Dermatol 2000; 136: 675-676

${ }^{31}$ Bagel J, Garland WT, Breneman D, Holick M, Littlejohn TW, Crosby D, Faust $\mathrm{H}$, Fivenson D, Nichols J. Administration of $\mathrm{DAB}_{389} \mathrm{IL}-2$ to patients with recalcitrant psoriasis: a double-blind, phase II multicenter trial. J Am Acad Dermatol 1998; 38: 938 - 944
${ }^{32}$ Gottlieb SL, Gilleaudau P, Johnson R, Estes L, Woodworth TG, Gottlieb $A B$, Krueger JG. Response of psoriasis to a lymphocyte-selective toxin $\left(\mathrm{DAB}_{389} \mathrm{IL}-2\right)$ suggests a primary immune, but not keratinocyte, pathogenetic basis. Nature Medicine 1995; 1: $442-447$

${ }^{33}$ Gottlieb AB, Lebwohl M, Shirin S, Sherr A, Gilleaudeau P, Singer G, Solodkina G, Grossman R, Gisoldi E, Phillips S, Neisler HM, Krueger JG. Anti-CD4 monoclonal antibody treatment of moderate to severe psoriasis vulgaris: results of a pilot, multicenter, multiple-dose, placebocontrolled study. J Am Acad Dermatol 2000; 43: 595-604

${ }^{34}$ Branco L, Barren P, Mao SY, Pfarr D, Kaplan R, Postema C, Langermann $\mathrm{S}$, Koenig S, Johnson S. Selective deletion of antigen-specific, activated T cells by a humanized MAB to CD2 (MEDI-507) is mediated by NK cells. Transplantation 1999; 68: 1588-1596

35 Bashir SJ, Maibach HI. Alefacept (Biogen). Curr Opin Investig Drugs 2001; 2: 631-634

${ }^{36}$ Krueger GG, Papp KA, Stough DB, Loven KH, Gulliver WP, Ellis CN. A randomized, double-blind, placebo-controlled phase III study evaluating efficacy and tolerability of 2 courses of alefacept in patients with chronic plaque psoriasis. J Am Acad Dermatol 2002; 47: 821 - 833

${ }^{37}$ Gottlieb AB, Krueger JG, Wittkowski K, Dedrick R, Walicke PA, Garovoy M. Psoriasis as a model for T-cell-mediated disease: immunobiologic and clinical effects of treatment with multiple doses of efalizumab, an anti-CD11a antibody. Arch Dermatol 2002; 138: 591 -600

${ }^{38}$ Cather JC, Cather JC, Menter A. Modulating T cell responses for the treatment of psoriasis: a focus on efalizumab. Expert Opin Biol Ther 2003; 3: $361-370$

${ }^{39}$ Schön MP, Krahn T, Schön M, Rodriguez ML, Antonicek H, Schultz JE, Ludwig RJ, Zollner TM, Bischoff E, Bremm KD, Schramm M, Henninger K, Kaufmann R, Gollnick HP, Parker CM, Boehncke WH. Efomycine M, a new specific inhibitor of selectin, impairs leukocyte adhesion and alleviates cutaneous inflammation. Nat Med 2002; 8: 366- 372

${ }^{40} \mathrm{Ghoreschi} \mathrm{K}$ et al. Interleukin-4 therapy of psoriasis induces Th2 responses and improves human autoimmune disease. Nat Med 2003; 9: $40-46$

${ }^{41}$ Asadullah K, Docke WD, Sabat RV, Volk HD, Sterry W. The treatment of psoriasis with IL-10: rationale and review of the first clinical trials. Expert Opin Investig Drugs 2000; 9: 95-102

42 Friedrich M, Docke WD, Klein A, Philipp S, Volk HD, Sterry W, Asadullah K. Immunomodulation by interleukin-10 therapy decreases the incidence of relapse and prolongs the relapse-free interval in psoriasis. J Invest Dermatol 2002; 118: 672-677

43 Trepicchio WL, Ozawa M, Walters IB, Kikuchi T, Gilleaudeau P, Bliss JL, Schwertschlag U, Dorner AJ, Krueger JG. Interleukin-11 therapy selectively downregulates type I cytokine proinflammatory pathways in psoriasis lesions. J Clin Invest 1999; 104: 1527-1537 